\title{
DESENVOLVIMENTO DE APLICATIVO EDUCACIONAL AUXILIAR EM PYTHON PARA AS DISCIPLINAS DE MECÂNICA DOS FLUIDOS E HIDRÁULICA
}

\author{
João Lucas Melo de Oliveira-joao_lucasmo@hotmail.com \\ Centro Universitário Senac - Santo Amaro \\ Avenida Engenheiro Eusébio Stevaux, 823 \\ 04696-000 - São Paulo - São Paulo
}

\author{
Alexandre Saron-alexandre.saron@sp.senac.br \\ Centro Universitário Senac - Santo Amaro \\ Avenida Engenheiro Eusébio Stevaux, 823 \\ 04696-000 - São Paulo - São Paulo
}

\begin{abstract}
Resumo: O presente trabalho teve como objetivo desenvolver um aplicativo educacional auxiliar em Python voltado para as disciplinas de Mecânica dos Fluidos e Hidráulica. $O$ aplicativo desenvolvido com a versão 3.8.3 - 64 bit do Python permite calcular e dimensionar parâmetros de projetos de instalações hidráulicas de recalque como perdas de carga por atrito, perdas localizadas, potência de bomba de recalque e NPSH disponível a partir da inserção de dados pelo usuário. Esses dados são informações básicas encontradas nos exercícios e problemas sobre esse tema, como temperatura, vazão do projeto, material da tubulação, extensão da tubulação, altura de sucção, altura de recalque, entre outros. $O$ aplicativo possui potencial como ferramenta educacional para implantação de tecnologias e uso de laboratórios de Informática nos cursos de engenharia, como preconizado pelas novas Diretrizes Curriculares Nacionais dos cursos de engenharia.
\end{abstract}

Palavras-chave: Mecânica dos Fluidos. Hidráulica. Aplicativo educacional. Python.

\section{INTRODUÇÃO}

Com o passar do tempo e com a evolução tecnológica, as metodologias de ensinoaprendizagem passam por mudanças de forma a acompanhar essa evolução, uma vez que o mercado e a sociedade exigem cada vez mais profissionais capacitados e competentes que tenham acompanhado tal evolução. No contexto da engenharia, a informática está cada vez mais presente, desde os programas de desenho auxiliado por computador até aplicativos do tipo "calculadora", que desempenham a função de realizar cálculos trabalhosos e complexos apenas com os dados inseridos pelo usuário. Essa é uma área já consolidada da pesquisa em educação, porém em constante evolução e que exige que a formação de professores e alunos contemplem a utilização de programas e o computador como objeto para estudo (MAIA; BARRETO, 2012; OLIVEIRA; FERREIRA; CRUZ, 2019).

Segundo Valente (1993), a implantação da informática na educação consiste de quatro ingredientes: "o computador, o software educativo, o professor capacitado para usar o computador como ferramenta educacional e o aluno". De acordo com o autor, o software educacional é tão importante quanto os outros ingredientes, pois sem ele não há possibilidade do uso do computador e da informática na educação.

Assim, o presente artigo tem como objetivo demonstrar como um aplicativo educacional desenvolvido com a linguagem Python pode auxiliar o processo de ensino-aprendizagem no âmbito das disciplinas de Mecânica dos Fluidos e Hidráulica, ou Fenômenos de Transporte 
em alguns cursos, para dimensionamento de instalações de recalque de água, perdas de carga e potência de bomba de recalque. Ainda possibilita a integração e aplicação de outras disciplinas tidas como básicas para os cursos de engenharia, independente da habilitação específica, como Matemática e Algoritmos e Programação. A integração entre disciplinas, o uso de laboratório de Informática e o desenvolvimento de competências relacionadas à tecnologia estão previstas nas novas Diretrizes Curriculares Nacionais para os cursos de engenharia, através da Resolução 02 de 24 de abril de 2019 (BRASIL, 2019). O aplicativo a ser apresentado pode ser classificado, de acordo com Valente (1993), como "Aplicativo para uso do professor e do aluno" por se tratar de um aplicativo do tipo calculadora, semelhante a proposta apresentada por Oliveira, Ferreira e Cruz (2019) para o dimensionamento de estruturas de concreto armado.

Para obter o código fonte do aplicativo desenvolvido enviar e-mail para o autor com a solicitação.

\section{FUNDAMENTAÇÃO TEÓRICA}

Neste item serão apresentados conceitos teóricos relacionados ao funcionamento do aplicativo, que realiza o dimensionamento de perdas de carga, potência de bomba e NPSH disponível para instalações hidráulicas de recalque.

\subsection{Características da água}

Há duas características importantes da água que são consideradas nesses cálculos: a viscosidade dinâmica e a pressão de vapor, as duas em função da temperatura. Os gráficos de variação da viscosidade dinâmica e da pressão de vapor da água em função da temperatura estão apresentados nas Figuras 1 e 2, elaborados a partir de dados de Netto, et al. (1998) e com as respectivas equações ajustadas, que foram incorporadas ao código do aplicativo.

Figura 1 - Viscosidade dinâmica da água em função da temperatura.

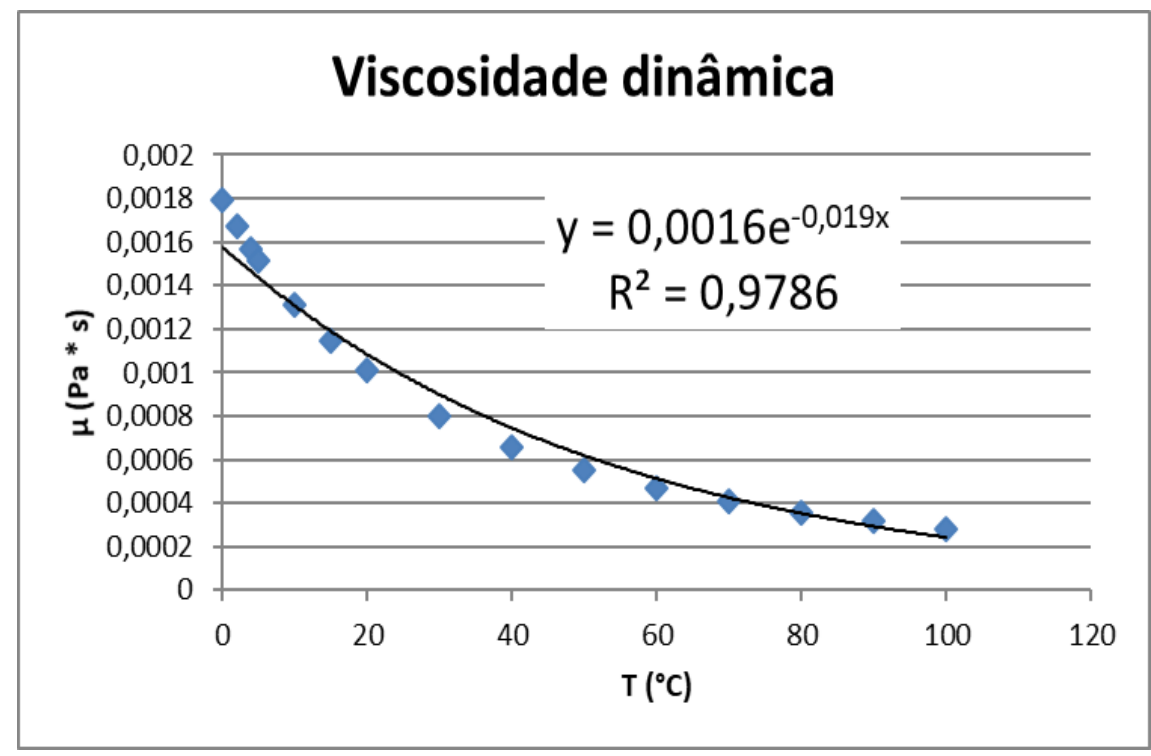

Fonte: adaptado de Netto, et al., 1998. 
Figura 2 - Pressão de vapor da água em função da temperatura.

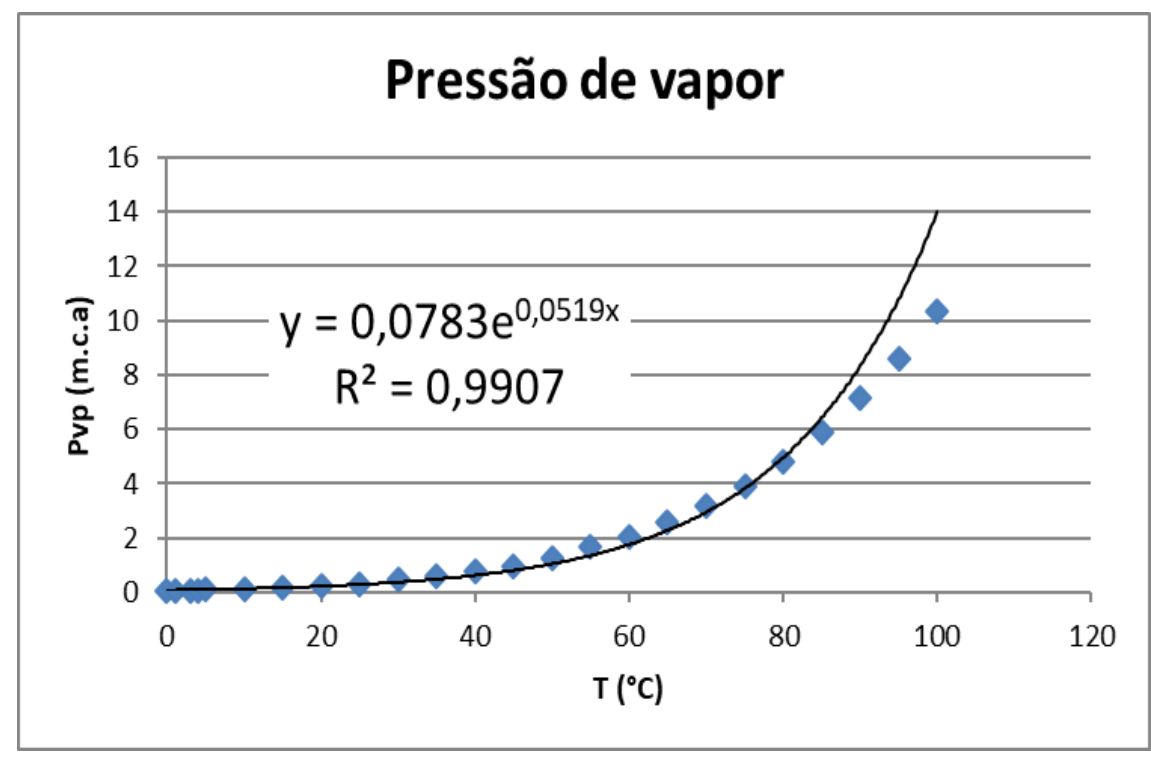

Fonte: adaptado de Netto, et al., 1998.

No aplicativo em questão não foi considerada a variação de massa específica da água, pois esta sofre uma variação desprezível na faixa de temperatura em que o aplicativo pode realizar seus cálculos (de 0 a $\left.100^{\circ} \mathrm{C}\right)$ (NETTO, et al., 1998).

\subsection{Perda de carga por atrito}

Para se calcular a perda de carga por atrito em tubulações é utilizada a fórmula de DarcyWeisbach, apresentada na Equação (1):

$$
h f=\frac{L}{D} \frac{V^{2}}{2 g} f
$$

Onde hf representa a perda de carga $(\mathrm{m}), \mathrm{L}$ é a extensão total da tubulação $(\mathrm{m}), \mathrm{D}$ é o diâmetro da tubulação $(\mathrm{m}), \mathrm{V}$ é a velocidade do escoamento $\left(\mathrm{m} \mathrm{s}^{-1}\right)$, g é a aceleração da gravidade $\left(9,8 \mathrm{~m} \mathrm{~s}^{-2}\right)$ e f é o fator de atrito, termo adimensional e que depende da rugosidade do material da tubulação, diâmetro e número de Reynolds. O número de Reynolds é calculado de acordo com a Equação (2):

$$
R e=\frac{V D \rho}{\mu}
$$

Onde Re é o número de Reynolds (adimensional), $\rho$ é a massa específica do fluido (no caso da água considera-se $1000 \mathrm{~kg} \mathrm{~m}^{-3}$ ), e $\mu$ é a viscosidade dinâmica do fluido ( $\mathrm{Pa} \mathrm{s}$ ). Se o número de Reynolds for menor que 2300, o regime de escoamento é considerado laminar e o fator de atrito pode ser calculado pela Equação (3):

$$
f=\frac{64}{R e}
$$

Para número de Reynolds entre 2300 e 4000 o regime de escoamento é considerado transiente, e para Reynolds maior que 4000 o regime de escoamento é considerado turbulento. Em ambos os casos é necessário utilizar o diagrama de Moody para se obter o fator de atrito em função do número de Reynolds e a rugosidade relativa da tubulação, dada pela razão entre a rugosidade do material da tubulação e seu diâmetro (WHITE, 2011). A Figura 3 apresenta o diagrama de Moody. 
Figura 3 - Diagrama de Moody.

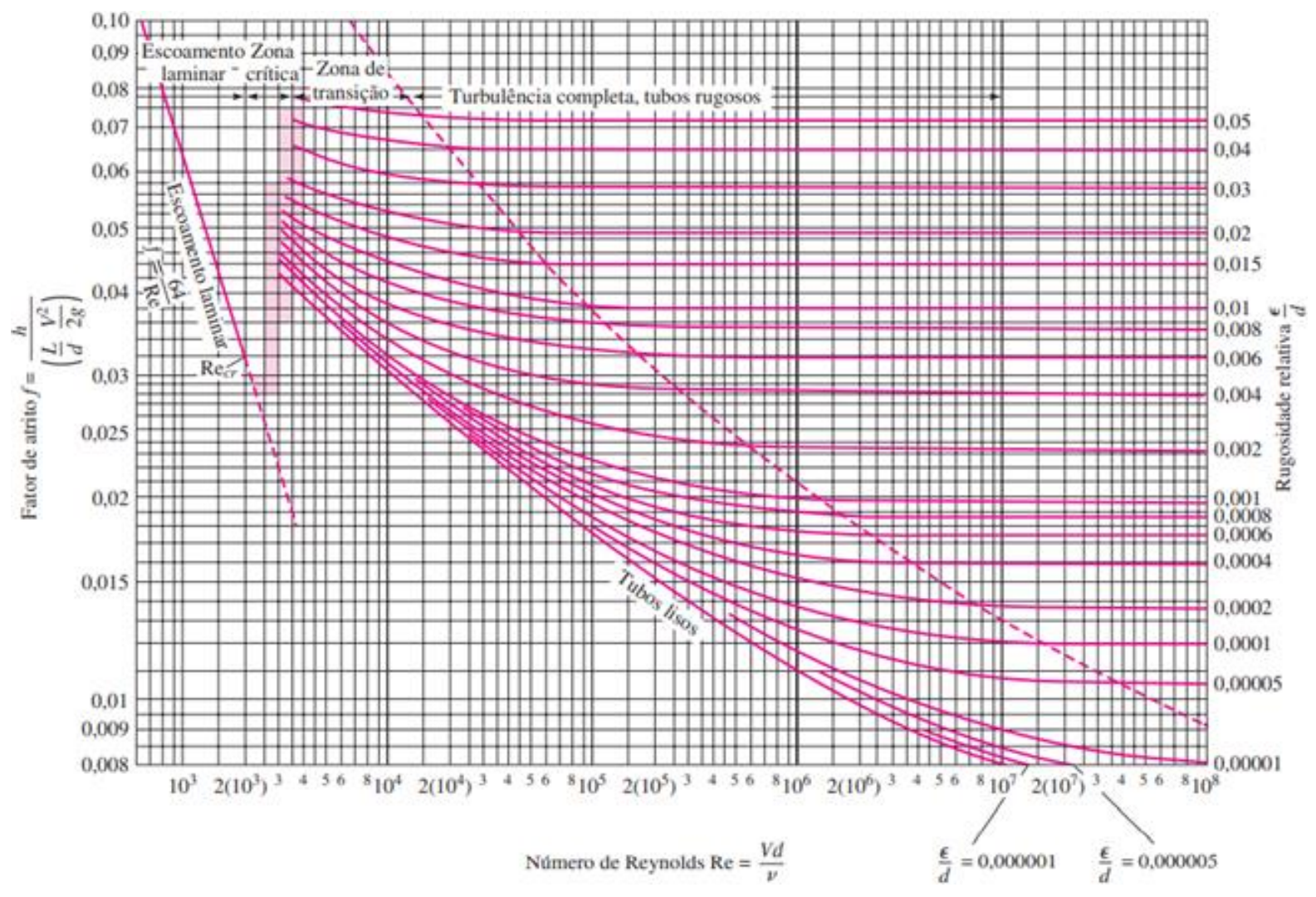

Fonte: White, 2011.

A Tabela 1 apresenta os valores de rugosidade $(\epsilon)$ de alguns materiais utilizados em tubulações.

Tabela 1 - Rugosidade de materiais utilizados em tubulações.

\begin{tabular}{c|c}
\hline Material & Rugosidade $\boldsymbol{\epsilon}(\mathbf{m m})$ \\
\hline Aço rebitado & 9 \\
Concreto & 3 \\
Madeira arqueada & 0,9 \\
Ferro fundido & 0,26 \\
Ferro galvanizado & 0,15 \\
Aço trefilado & 0,0015 \\
Plástico ou vidro & 0 (tubos lisos) \\
\hline
\end{tabular}

Fonte: adaptado de Netto, et al., 1998 e White, 2011.

Alternativamente, pode ser utilizada a equação de Colebrook-White para obtenção do valor do fator de atrito de acordo com a Equação (4):

$$
\frac{1}{\sqrt{f}}=-2 \log \left(\frac{\epsilon}{3,7 D}+\frac{2,51}{R e \sqrt{f}}\right)
$$

A Equação (4) não possui solução algébrica, sendo solucionada com técnicas de Cálculo Numérico para o valor do fator de atrito. Assim, muitos autores se dedicaram a encontrar equações explícitas alternativas. Segundo Minhoni, et al. (2020), a equação proposta por Vatankhah (2018) possui um desvio de menos de 1\% em relação à Equação (4) e é apontada como a melhor alternativa entre as equações estudadas pelos autores. Por isso, essa foi a equação utilizada no aplicativo para o cálculo do fator de atrito, mostrada na Equação (5): 
(C) COBENGE

"Os desafios para formar hoje o engenheiro do amanhã"

$$
\frac{1}{f^{0,5}}=0,8686 \ln \left(\frac{0,3984 R e}{(0,8686 s) \frac{s-0,645}{s+0,39}}\right)
$$

Onde $s=0,12363 \operatorname{Re}\left(\frac{\epsilon}{D}\right)+\ln (0,3984 R e)$.

\subsection{Perdas localizadas ou singulares}

As perdas localizadas, também chamadas de singulares, são causadas pela passagem do fluido em acessórios, dispositivos e peças presentes na tubulação, como curvas, cotovelos, válvulas, registros, entre outros. A perda localizada causada por um dispositivo é calculada pela Equação (6):

$$
h L=K \frac{V^{2}}{2 g}
$$

Onde hL é a perda localizada (m), K é um coeficiente empírico adimensional, obtido experimentalmente. A Tabela 2 apresenta os valores do coeficiente $\mathrm{K}$ para alguns dispositivos utilizados em tubulações e presentes no aplicativo desenvolvido.

Tabela 2 - Coeficiente empírico de perda localizada para alguns dispositivos.

\begin{tabular}{c|c|c|c}
\hline Dispositivo & Coeficiente K & Dispositivo & Coeficiente K \\
\hline Cotovelo $90^{\circ}$ & 0,9 & Registro de gaveta & 0,2 \\
Cotovelo 45 & 0,4 & Registro de ângulo & 5 \\
Curva 90 & 0,4 & Registro de globo & 10 \\
Curva 45 & 0,2 & Pé de crivo & 10 \\
Curva 22,5 & 0,1 & Válvula de retenção & 2,5 \\
Controlador de vazão & 2,5 & Medidor Venturi & 2,5 \\
Passagem bilateral & 1,8 & & \\
(Tê) & & & \\
\hline
\end{tabular}

Fonte: adaptado de Netto, et al., 1998 e White, 2011.

\subsection{Potência da bomba de recalque}

Para o cálculo da potência da bomba é utilizada a Equação (7):

$$
P=\frac{Q \rho g\left(h_{\text {geométrica }}+h f_{\text {total }}+h L_{\text {total }}\right)}{n_{\text {bomba }} n_{\text {motor }} 735}
$$

Onde $\mathrm{P}$ é a potência da bomba (cv), Q é a vazão do projeto $\left(\mathrm{m}^{3} \mathrm{~s}^{-1}\right), \rho$ é a massa específica do fluido, g é a aceleração da gravidade, $h_{\text {geométrica }}$ é a altura geométrica, ou estática, total do projeto, $h f_{\text {total }}$ é a perda de carga por atrito total, $h L_{\text {total }}$ é a perda localizada total, $n_{\text {bomba }}$ é o rendimento da bomba (\%) e $n_{\text {motor }}$ é o rendimento do motor da bomba (\%). A altura geométrica do projeto deve ser cuidadosamente calculada levando-se em consideração se a bomba está afogada (Figura 4) ou não (Figura 5), ou seja, se há coluna de água acima ou abaixo da linha da bomba, pois isto influencia o cálculo da potência da bomba (NETTO, et al., 1998). 


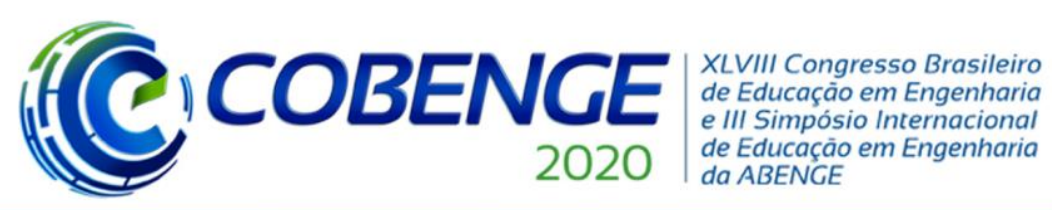

"Os desafios para formar hoje o engenheiro do amanhã"

\section{Evento On-line}

Figura 4 - Bomba afogada.

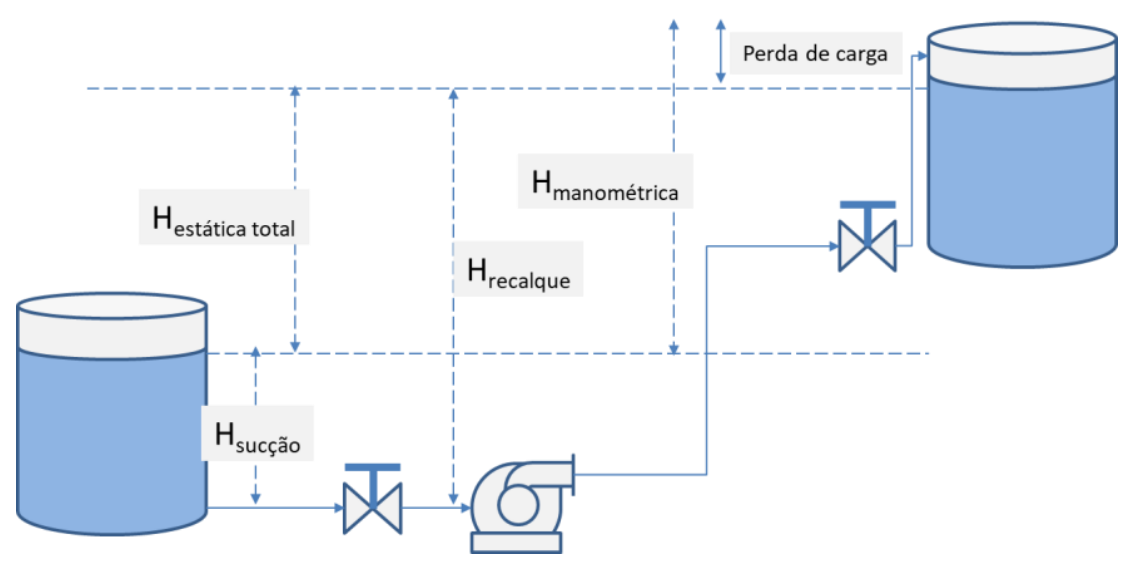

Fonte: autoria própria.

Figura 5 - Bomba não afogada.

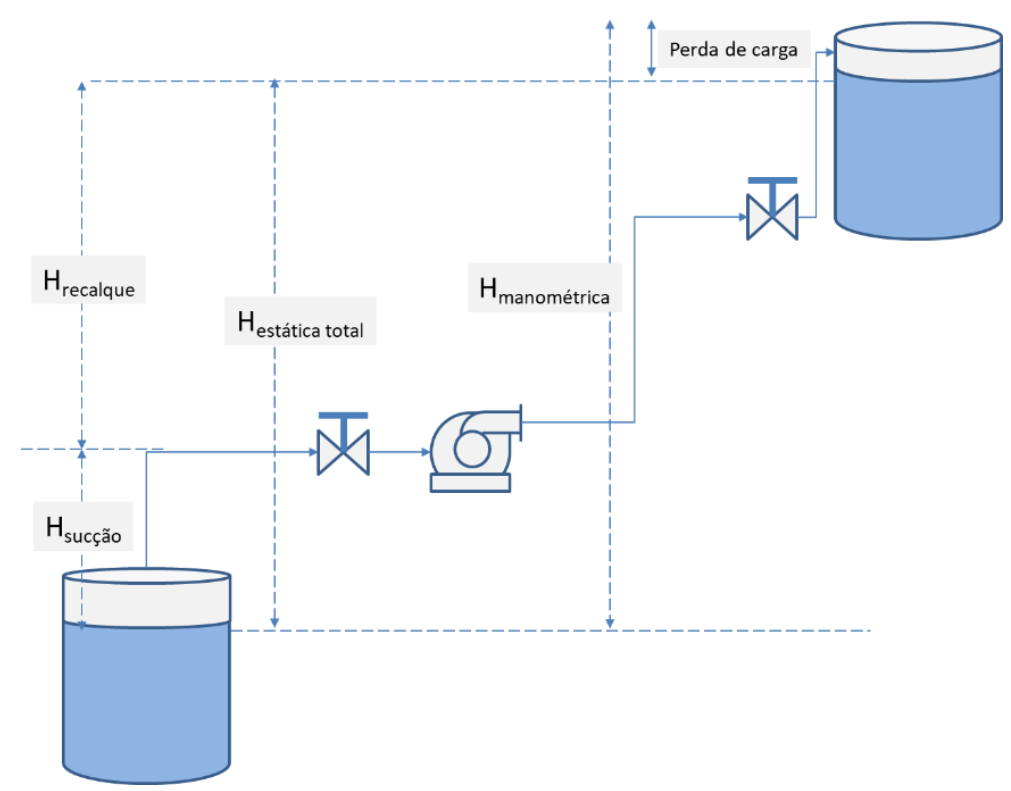

Fonte: autoria própria.

\subsection{NPSH disponível}

NPSH (Net Positive Suction Head) disponível, em português significa energia no líquido na entrada da bomba, é a quantidade de energia, em m, disponível na sucção, ou seja, a carga positiva e efetiva na sucção. O NPSH disponível é calculado de acordo com a Equação (8):

$$
N P S H_{\text {disponível }}=P s+P a-P v p-P f
$$

Onde Ps é a pressão exercida pela coluna de líquido acima da linha da bomba (m), Pa é a pressão absoluta exercida sobre a superfície do líquido no tanque de sucção (m), Pvp é a pressão de vapor (m), e Pf é a perda de carga total na linha de sucção (antes da bomba), representada pela soma das perdas por atrito e as perdas localizadas, em $\mathrm{m}$. O NPSH disponível deve ser maior que o NPSH requerido (informado pelo fabricante da bomba) para que a bomba funcione sem problemas e evitando problemas como a cavitação (formação de bolsões de ar dentro da bomba) (NETTO, et al., 1998). 
(C) COBENGE

"Os desafios para formar hoje o engenheiro do amanhã"
$\mathrm{Ol} \mathrm{a} \mathrm{O} 3$ de dezembro Evento On-line

\section{DESCRIÇÃO E FUNCIONAMENTO DO APLICATIVO}

O aplicativo consiste de uma única interface gráfica com seis grandes campos, divididos em perdas por atrito na linha de sucção e de recalque, perdas localizadas na linha de sucção e recalque, potência da bomba de recalque e NPSH disponível. A Figura 6 apresenta a interface gráfica do aplicativo desenvolvido.

Figura 6 - Interface gráfica do aplicativo desenvolvido.

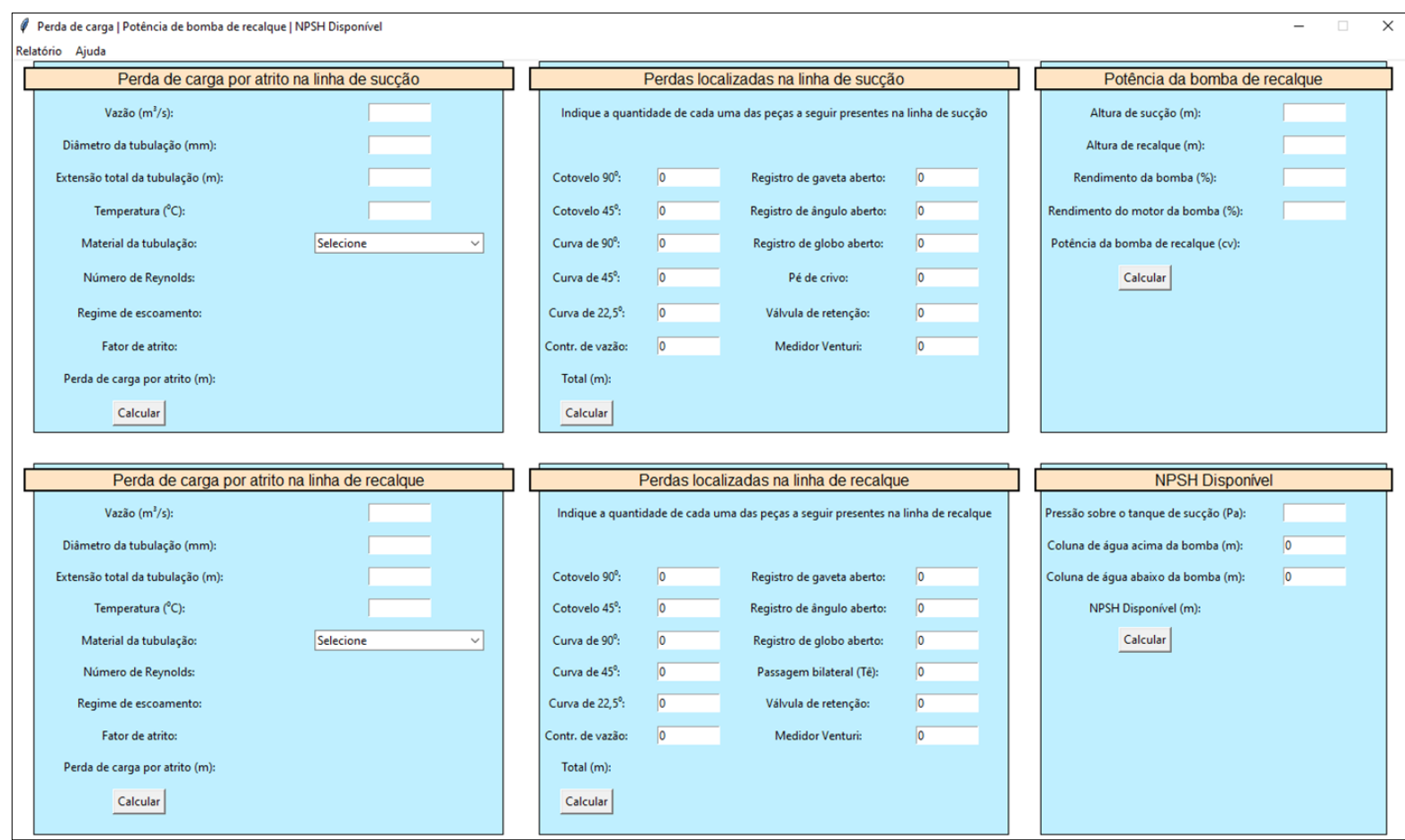

Fonte: autoria própria.

O aplicativo conta ainda com uma barra de menu onde podem ser encontradas informações básicas sobre como utilizar os campos e a função de "Gerar relatório". Após os cálculos é possível gerar um relatório em formato .txt que estará localizado na mesma pasta do arquivo do aplicativo. A Figura 7 apresenta as funções e informações disponíveis na barra de menu.

Figura 7 - Funções e informações presentes na barra de menu do aplicativo.

\begin{tabular}{|c|c|c|c|}
\hline \multirow{2}{*}{\multicolumn{2}{|c|}{ Perda de carga | Potência de bomba de reca }} & \multicolumn{2}{|c|}{ Perda de carga | Potência de bomba de recalque | NPSH Disponivel } \\
\hline & & Relatório & Ajuda \\
\hline Relatório & Ajuda & & Como utilizar o programa? \\
\hline \multirow{2}{*}{\multicolumn{2}{|c|}{ Gerar relatório }} & & O que é altura de \\
\hline & & & O que é coluna de água acima ou abaixo da linha da bomba? \\
\hline \multicolumn{2}{|c|}{ Informações sobre geração de relatório } & & $\begin{array}{l}\text { O que é pressão sobre o tanque de sucção? } \\
\text { O que é o NPSH Disponivel? }\end{array}$ \\
\hline
\end{tabular}

Fonte: autoria própria.

Para demonstração da funcionalidade do aplicativo, serão utilizados dados adaptados de um exercício de Vilanova (2011). O Quadro 1 apresenta os dados do exercício utilizado para demonstração. 
Quadro 1 - Dados do exercício a ser utilizado para demonstração da funcionalidade do aplicativo.

\begin{tabular}{|c|c|}
\hline Dado & Valor \\
\hline Temperatura $\left({ }^{\circ} \mathrm{C}\right)$ & 20 \\
\hline Vazão $\left(\mathrm{m}^{3} \mathrm{~s}^{-1}\right)$ & 0,00075 \\
\hline Material da tubulação & Ferro galvanizado (rugosidade =0,15 mm) \\
\hline Extensão da tubulação de sucção $(\mathrm{m})$ & 6 \\
\hline Extensão da tubulação de recalque $(\mathrm{m})$ & 19 \\
\hline Diâmetro da tubulação $(\mathrm{mm})$ & 4 \\
\hline Cotovelos $90^{\circ}$ na linha de recalque & 1 \\
\hline Registro de globo na linha de recalque & 1 \\
\hline Registro de gaveta na linha de recalque & 1 \\
\hline Cotovelo 90 ${ }^{\circ}$ na linha de sucção & 1 \\
\hline Pé de crivo na linha de sucção & 3 \\
\hline Altura de sucção $(\mathrm{m})$ & 3 \\
\hline Altura de recalque $(\mathrm{m})$ & 75 \\
\hline Rendimento da bomba $(\%)$ & 75 \\
\hline Rendimento do motor da bomba $(\%)$ & 0 \\
\hline Pressão sobre o tanque de sucção $(\mathrm{Pa})$ & Tanque aberto $=$ pressão atmosférica $=101325$ Pa \\
\hline Coluna de água acima da linha da bomba $(\mathrm{m})$ & $-1,5$ \\
\hline $\begin{array}{c}\text { Coluna de água abaixo da linha da bomba }(\mathrm{m}) . \\
\text { Caso haja coluna de água abaixo da linha da } \\
\text { bomba deve ser inserido um valor negativo. }\end{array}$ & \\
\hline
\end{tabular}

Fonte: adaptado de Vilanova, 2011.

A Figura 8 apresenta os resultados obtidos do exercício utilizando o aplicativo.

Figura 8 - Resultados do exercício de demonstração proposto.

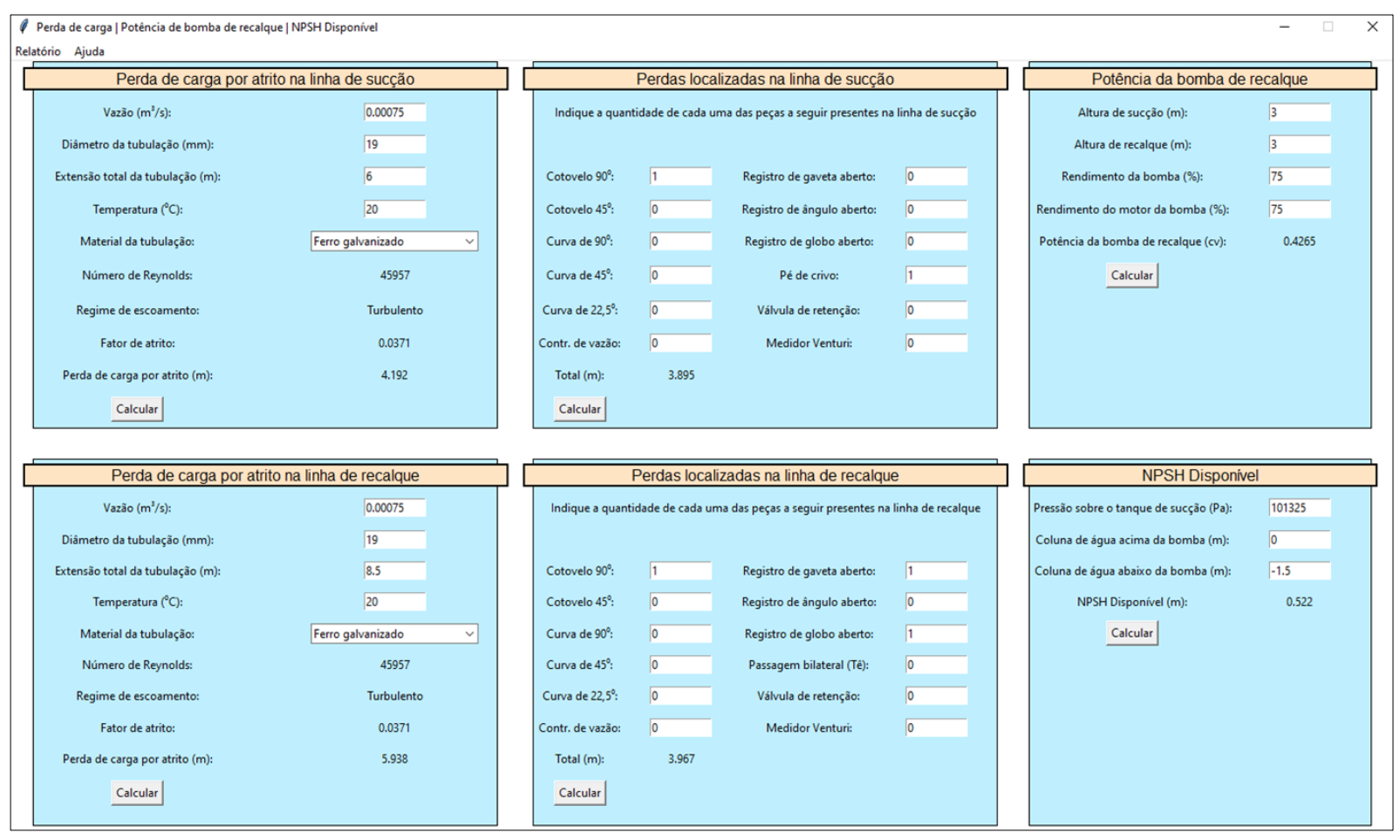

Fonte: autoria própria. 


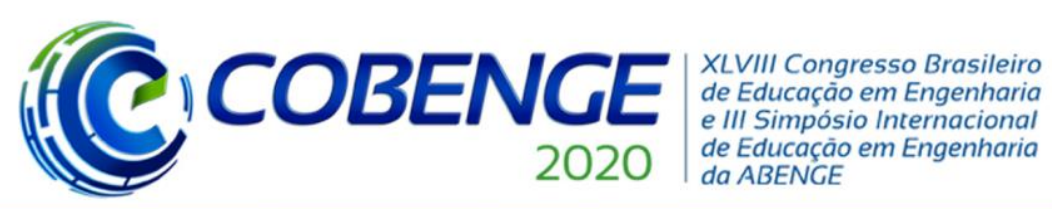

"Os desafios para formar hoje o engenheiro do amanhã"

A Figura 9 apresenta o relatório gerado para os resultados apresentados na Figura 8.

Figura 9 - Relatório gerado pelo aplicativo.

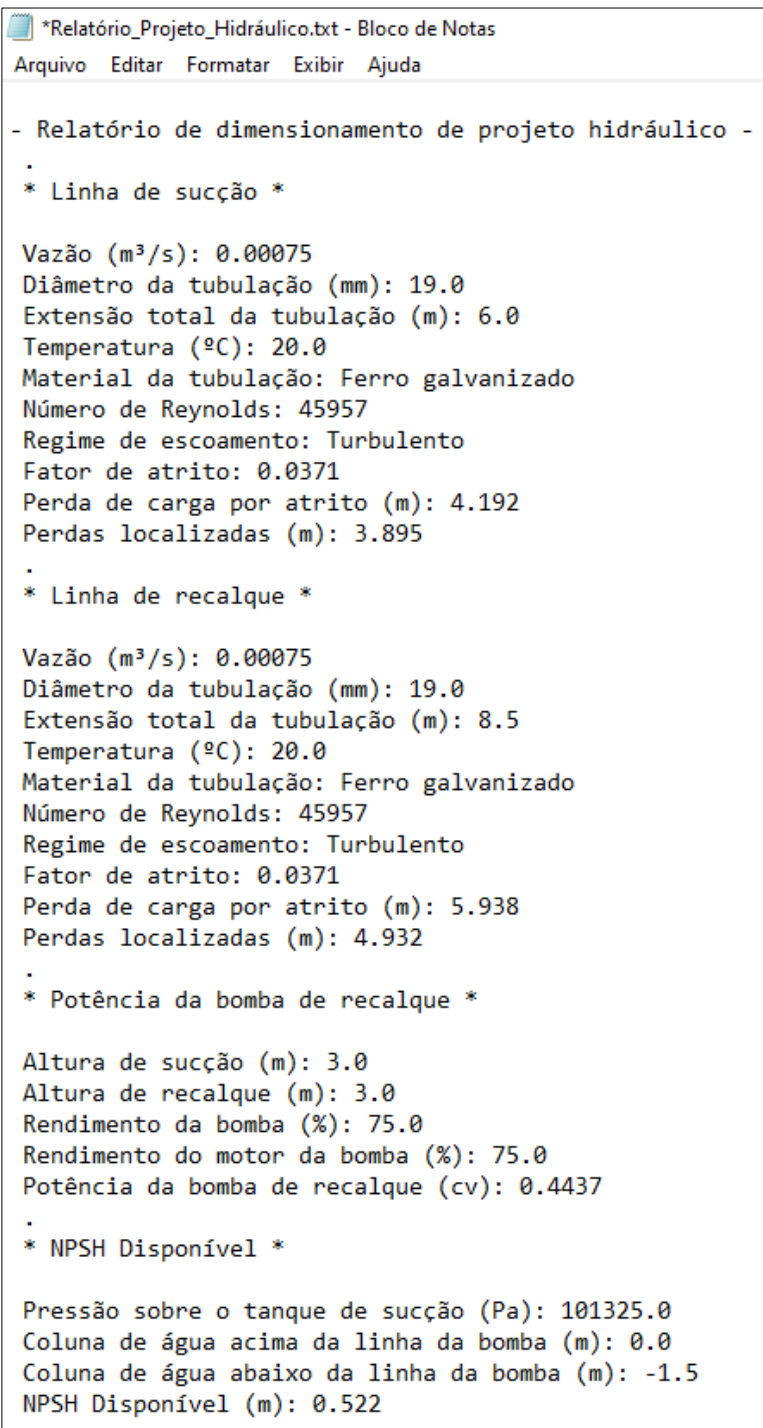

Fonte: autoria própria.

\section{CONCLUSÃO}

O aplicativo desenvolvido e aqui apresentado se mostra uma potencial ferramenta educacional auxiliar para as aulas de Mecânica dos Fluidos e Hidráulica que pode ser utilizada para validação de resultados obtidos por cálculos feitos à mão pelos estudantes, com resultados semelhantes àqueles alcançados por Araújo, Bezerra e Tavares (2018) com proposta semelhante à do presente artigo. Além disso, é possível ampliar os estudos em cima de um aplicativo educacional como este ao aprofundar os conceitos de Algoritmos e Programação envolvidos em sua criação e utilizá-lo como objeto de estudo nesta disciplina também, mostrando aplicações dos conceitos de lógica, algoritmos e linguagem de programação aplicados à resolução de problemas de engenharia e economia de tempo com cálculos extensos e trabalhosos. Ressalta-se a importância da utilização de softwares e laboratório de Informática em cursos de engenharia como previsto pelas novas Diretrizes Curriculares Nacionais dos cursos de engenharia e também como desenvolvimento de habilidades e competências. 
"Os desafios para formar hoje o engenheiro do amanhã"

\section{Agradecimentos}

Prof. Dr. Eduardo Antonio Licco e Prof. Esp. Valdnei Dias Batista.

\section{REFERÊNCIAS}

ARAÚJO, D. S. A.; BEZERRA, J. E. C. D.; TAVARES, P. R. L. AppHidráulica - software para resolução de problemas de hidráulica. In: Anais XXVIII Congresso Latinoamericano de Hidráulica, Buenos Aires, set., 2018.

BRASIL. MEC/CNE/CES. Resolução $\mathbf{n}^{0} 2$ de 24 de abril de 2019. Disponível em: http://www.in.gov.br/web/dou/-/resolu\%C3\%87\%C3\%83o-n\%C2\%BA-2-de-24-de-abrilde2019-85344528 Acesso em: 01/07/2020.

MAIA, D. L.; BARRETO, M. C. Tecnologias digitais na educação: uma análise das políticas públicas brasileiras. In: Educação, Formação \& Tecnologias, v. 5, no 1, p. 47-61, maio, 2012.

MINHONI, R. T. A., et al. The performance of explicit formulas for determining the DarcyWeisbach friction factor. In: Engenharia Agrícola, Jaboticabal, v. 40, no 2, p. 258-265, mar./abr., 2020.

NETTO, J. M. A., et al. Manual de Hidráulica. 8ª ed. São Paulo: Edgard Blücher, 1998.

OLIVEIRA, C. J.; FERREIRA, C. H.; CRUZ, J. F. Desenvolvimento de plataforma educacional voltada a disciplina de concreto armado. In: Anais XLVII COBENGE, Fortaleza/CE, 2019.

VALENTE, J. A. Diferentes usos do computador na educação. In: Em aberto, Brasília, ano 12, nº 57, jan./mar., 1993.

VILANOVA, L. C. Mecânica dos Fluidos. $3^{\text {a }}$ ed. Santa Maria: Colégio Técnico Industrial de Santa Maria, 2011.

WHITE, F. M. Mecânica dos Fluidos. 6ª ed. Porto Alegre: AMGH, 2011.

\section{DEVELOPMENT OF AUXILIARY EDUCATIONAL APPLICATION IN PYTHON FOR THE DISCIPLINES OF FLUID MECHANICS AND HYDRAULICS}

Abstract: The present work aimed to develop an auxiliary educational application in Python focused on the disciplines of Fluid Mechanics and Hydraulics. The application developed with python version 3.8.3 - 64 bit allows you to calculate and scale parameters of hydraulic installations projects such as frictional load losses, localized losses, heat pump power and NPSH available from user data entry. These data are basic information found in the exercises and problems on this topic, such as temperature, project flow, pipe material, pipe extension, suction height, repression height, among others. The application has potential as an educational tool for the implementation of technologies and the use of Computer Laboratories in engineering courses, as recommended by the new National Curriculum Guidelines of engineering courses.

Keywords: Fluid Mechanics. Hydraulics. Educational application. Python. 